\title{
Performance Evaluation of LTE network via using Fixed/Mobile Femtocells
}

\author{
Rand Raheem, Aboubaker Lasebae, Jonathan Loo \\ School of Science \& Technology, Middlesex University \\ London, United Kingdam
}

\{R.Raheem; A.Lasebae; J.Loo\} @ mdx.ac.uk

\begin{abstract}
This paper examines the concept of Mobile Femtocells to be the revolution of the next generation cellular networks. Mobile Femtocells can be deployed in public transportation vehicles such as trains, buses or private cars that form its own cell inside vehicles to serve vehicular and mobile User Equipments. The purpose of this study is to help cell-edge users to have better signal strength. Therefore, an investigation into Long Term Evolution cell-edge users' performance is being conducted by investigating the deployment of Mobile Femtocells in LTE system. The throughput for cell edge users can be improved by deploying Fixed/Mobile Femtocells. In this paper, two scenarios have been considered in the case of Fixed/Mobile Femtocells. The handover of Mobile Femtocell has been expressed in three more scenarios. The achieved results via Matlab simulation showed that Mobile Femtocells' users have enjoyed better Quality of Services than Fixed Femtocells' users. The improved performance has been noticed through the improvement of the Mobile Femtocells UEs' spectral efficiency, throughput and SINR over the Fixed Femtocells' users. The system behavior has been investigated under low, medium and high load traffic before and after adding the Mobile Femtocells. The results showed that adding the Mobile Femtocells in the high loaded traffic areas has the biggest affect on improving the UE's throughput.
\end{abstract}

Keywords-LTE, Femtocells, Mobile and Fixed Relays, OFDMA, outdoor and indoor environments, mobile femtocells \& fixed femtocells.

\section{INTRODUCTION}

The Long Term Evolution (LTE) has been standardised by the $3^{\text {rd }}$ Generation Partnership Project (3GPP). LTE is a standard for wireless communication of high speed data for mobile phones and data terminals. The need to have better spectral efficiency and data rate speed were the purpose behind developing LTE technology. A more intelligent BS (eNB) was introduced to simplify the system architecture and minimise the control and User Equipment (UE) plane latency. Moreover, LTE adapts Orthogonal Frequency Division Multiple Access (OFDMA) as the base technique for sharing resources among multiple UEs. 3GPP extended the original proposed LTE which is known as LTE-Advance that aims to data rates up to 3 Gbps and $1.5 \mathrm{Gbps}$ in Downlink (DL) and Uplink (UL) respectively by employing advanced multi-antenna multipleinput multiple-output (MIMO) techniques, carrier aggregation (CA) and other schemes.
However, in relay communication, intermediate nodes are used to relay the data from and to the eNB [1]. The first deployment of relay nodes was fixed then the need of deploying the mobile relays came which was the debut of Mobile Femtocells (MFemtos) [2]. The fixed relays are deployed at locations according to cell planning and radio optimisation to improve the users' throughput, expand the coverage and improve the cell edge users' performance [3]. While mobile relays are mobile wireless nodes that reach several remote areas which are out of the coverage area of the eNB [9]. Those mobile relays can be either; mobile user relays or mobile networks. In the mobile user relays, specific users will be chosen to work as mobile relays within close vicinity to relay the information from and to the eNB in LTE networks. On the other hand, the mobile (moving) network offers that relay nodes to be placed on moving vehicles e.g. trains, buses or private cars e.g. Taxis to receive and send data from/to eNB then forward it to the UEs in the edges of the LTE networks. Most of the previous works have been focusing on fixed relays [4], Fixed Femtocells (FFemto) [5] or mobile user relays [6]. There are only few researches undertaken recently for mobile (moving) networks; therefore, this paper considers the MFemto as a next generation revolution in LTE network.

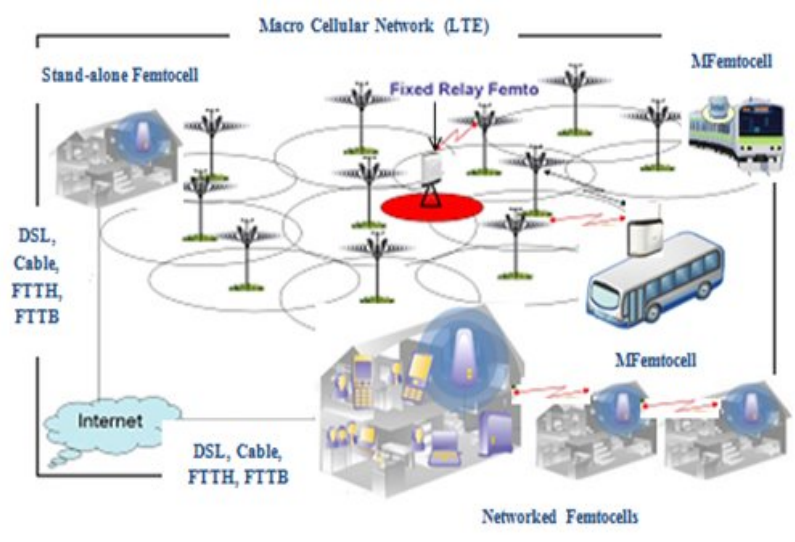

Fig.1. Fixed and Mobile Femtocell Technology

Figure 1 illustrates the fixed and mobile femtocells which could be either inside buildings, streets or public transportations like trains and buses. The MFemtos are moving hotspots with multiple UEs who are requesting diverse data services e.g. web browsing, e-mailing and video streaming. 
Since the UEs inside public or private vehicles may execute multiple handovers at the same time that cause significant increase in the signalling load and drop in the network connections. This leads to considering the MFemtos as a solution to minimise the signalling load, packet drops, handover $(\mathrm{HO})$ and time delay.

Femtocells are also known as home BSs or home evolved NodeB (Home eNB) [15]. Femtocells are small, low power and higher speed access points that users can install at home or camps to get better indoor coverage and improve the throughput of users with reducing the cost of implementation. In contrast, outdoor femtocells are placed outside buildings like streets or mountainous to increase the cell-edge users' performance in the cellular system. Femtocells can be connected to the operator's core network through the legacy broad band connection which might be an optical fiber or digital subscriber line. Since the transmission range between the femtocell and its users is short, the transmission power of users can be saved resulting in longer battery life.

The rest of this paper will be sectioned as following: Section II presents a background and discusses the related work. Section III gives an overview about MFemtos. Section IV demonstrates the considered Fixed \& Mobile Femtocells' scenarios while section $\mathrm{V}$ discusses some of the simulated results and finally section VI concludes this paper.

\section{RELATED WORK}

The need to improve the Quality of Service (QoS) of celledge users played an important role in developing the MFemto technology. This technology has the ability to cover the unknown dead spots that hard to be covered by the eNB in LTE networks so because of that MFemtos become the interest of many researches. However, several challenges can be faced with deploying the MFemtos which are needed to be considered from different aspects such as; sharing the resources between the macrocell and MFemto users, the spectral efficiency, consumption power, throughput, Signal Interference plus Noise Ratio (SINR), Signal to Noise Ratio (SNR) and Hanover (HO) procedure. Also, the main concern will be about developing a new technique that serves the largest possible number of users with concerning about improving the performance of cell-edge users. Both of the user selection and the resource allocation scheme are needed to be investigated in this study. Moreover, the position of the FFemtos and MFemtos is needed to be chosen wisely in order to have the smallest possible number of femtocells but with the largest possible coverage area. Using this technique reduces the implementation cost and interference so it is necessary to specify the cell threshold area and place the femtocells in this area.

More studies have been done by [8] on the resource management of femtocell, spectrum sharing efficiency in femtocell [8] and multi-hopping [4] in OFDMA based on LTE network [11].

It is known that femtocells can either be deployed indoors or outdoors [7] and it may share the same spectrum with the macrocell or utilise a specific spectrum which can be known as non- orthogonal mode and orthogonal mode respectively [8].
The difference between using the non-orthogonal and orthogonal mode is that in an orthogonal mode, the femtocells use a separate spectrum band that has not been used by the eNB which can have the advantage of avoiding interference from/to the macro cell, while in the non-orthogonal mode the femtocell may share the same spectrum with the macrocell.

Several studies have considered the interference issue and the impact of that on the network performance in LTE and LTE-Advanced network [10].

More researches have been done on the mobile user relays and the advantages of using this technology to improve the cell edge users' throughput [11]. Most of the achieved results showed that there is always an issue with the mobile users relay battery life since it is limited and cannot relayed upon when deploying large number of users. While, there are few studies that consider mobile relay nodes as mobile networks [12]. However, the issue with the mobile relay is the limited coverage area which is restricted to a few meters and the limited number of users served. Although, the limited coverage area can be the solution to the interference issue it raises other issues like the unnecessary $\mathrm{HO}$ issue and the HO time delay.

\section{MFEMTOCELL}

The concept of the MFemto has been derived from combining the concept of two technologies; the FFemto technology and the mobile relays. The main advantage of using MFemto is the ability of this small cell to be moved around and dynamically change its connection to the operator's core network. The concept of the MFemto can be seen as a practical implementation of mobile networks which can be deployed on public transport like trains and buses or on private cars.

The MFemto adapts the LTE's standard radio interface to communicate with the serving eNB and the group of UEs who are within the coverage of that particular MFemto. Several advantages have been addressed in the existing studies that potentially benefit the cellular networks as the following;

- Firstly, MFemtos have the ability to improve the spectral efficiency of the entire networks as the mobile relay stations.

- Secondly, MFemtos have the ability to reduce the signalling overhead for different network operations by performing the $\mathrm{HO}$ activities for UEs within the MFemtos coverage areas. Also, MFemto plays an important role in saving the battery life of UEs within the MFemto coverage area as they only involve in a short range communication which consumes less power and energy, instead of the long distance to the eNBs.

- Thirdly, the implantation of the MFemto is compatible with LTE system and there is no necessity to do any changes in the Radio Access Network (RAN).

- Fourthly, the MFemto traffic over the backhaul link is served like a regular user since there is a need only for a normal femtocell with special advanced capabilities to be able to move around and serve UEs in different 
areas within the coverage area of that particular MFemto. Also, MFemto has the flexibility to add more than one antenna and different antenna patterns to enjoy special diversity and beam-forming. Fifthly, UEs need a normal $\mathrm{HO}$ process to switch from the Macrocell (eNB) to the MFemto and vice versa since they are seeing the MFemto as a regular BS. The following figure illustrates the MFemto architecture in LTE system:

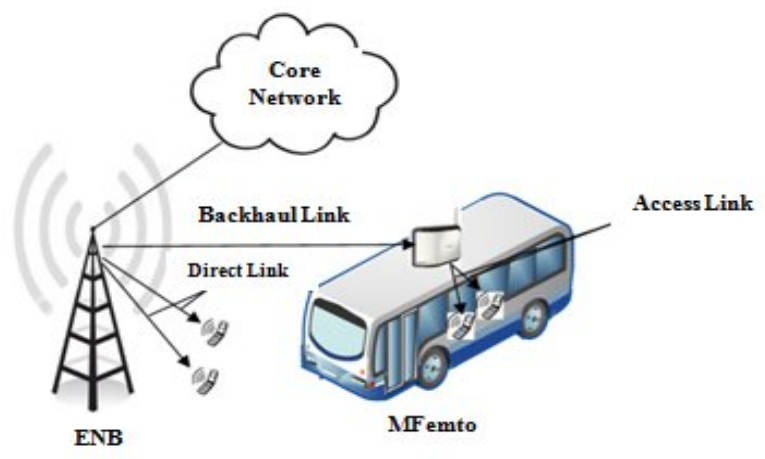

Fig.2. MFemto architecture

Figure 2 shows that there are three types of links which will be used to differentiate between the eNB-MFemto, MFemtoUE and eNB-UE links; the backhaul, access link and direct link respectively.

\section{FFEMTOCELL \& MFEMTOCELL SCENARIOS}

In this section several scenarios will be discussed in order to compare between FFemto and MFemto UEs in term of their UEs' performance. There are other scenarios have been disscused in [16].

$>$ Fixed Femto-Mobile UEs scenario

Placing the FFemtos near to the threshold of the cell will be the solution to improve the performance of the mobile cell edge UEs as the following figure shows;

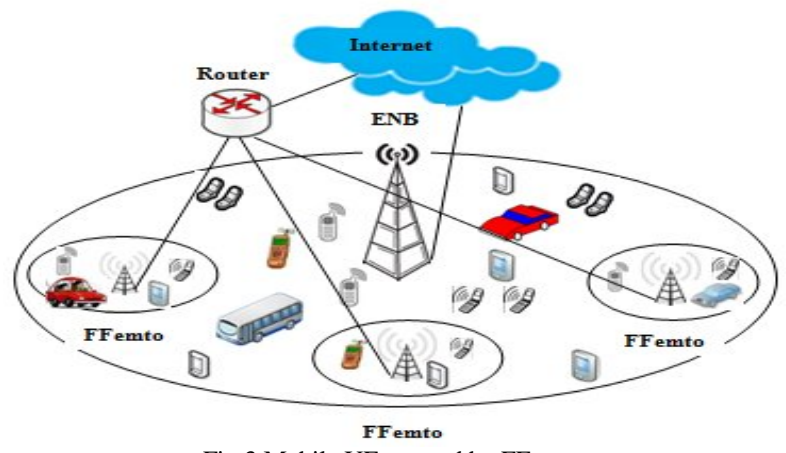

Fig.3.Mobile UEs served by FFemto

$>$ MFemto-Mobile UE scenario

This scenario can be represented when there is a bus full of vehicular UEs or a train full of mobile UEs. In this case several criteria will be considered e.g. UEs/MFemto speed, direction and distance. However, it is important to be mentioned here that this MFemto will be considered as a fixed femtocell for those UEs who are inside the bus while it will be considered as a mobile femtocell for those UEs who are outside the bus [16].

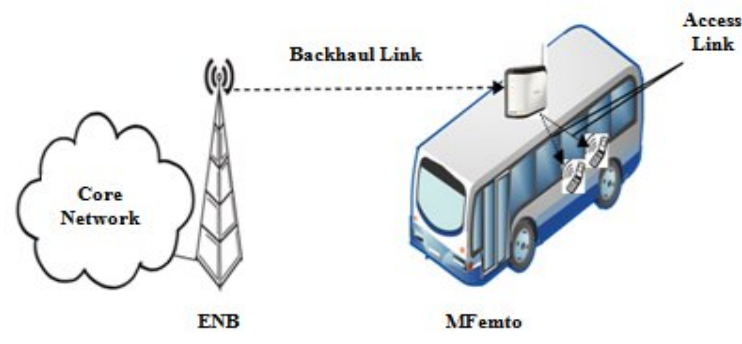

Fig.4.Mobile UEs with MFemto

$>$ MFemto HO scenarios

In this paper two HO scenarios will be considered that will lead later on to a third scenario. The first scenario is the HO from one MFemto to another close by MFemto and the second scenario is handing-over the MFemto from one eNB to another neighbouring eNB. Figure 5 illustrates when UEs move from train A to train B which means from one MFemto to another so the HO procedure needs to be established in order to maintain the signal strength of those moving out/in UEs.

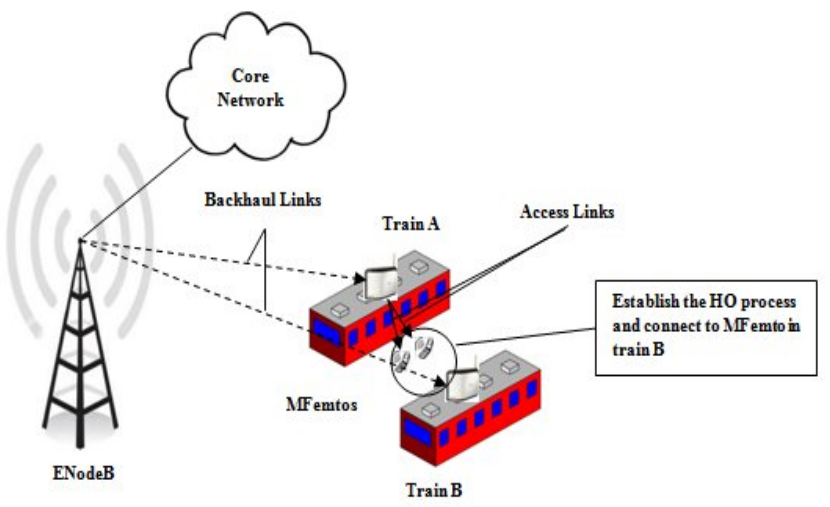

Fig.5.HO in MFemtos

The HO from one MFemto to another MFemto occurs when the UE needs to move out of the range of one MFemto to another. In this case the UE needs to select an appropriate target MFemto among many neighbouring MFemtos in order to be handed over to. Checking the authorisation of the UE during the $\mathrm{HO}$ preparation phase is an important part for the $\mathrm{HO}$ to be completed correctly.

The second scenario of the MFemto $\mathrm{HO}$ is when the MFemto moves away from one cell to another so in this case the MFemto needs to be handed over to the next neighbouring cell. In order to avoid the overload that the next cell might suffer from; another MFemto needs to be moved out from the second cell to another next neighbouring cell. In this scenario there should be an excellent time management otherwise the connection will go down. So whenever MFemto (A) starts its $\mathrm{HO}$ procedure; MFemto (B) needs to starts its $\mathrm{HO}$ procedure 
almost at the same time to move out of cell (B) to cell (C). Figure 6 shows how this scenario functions;

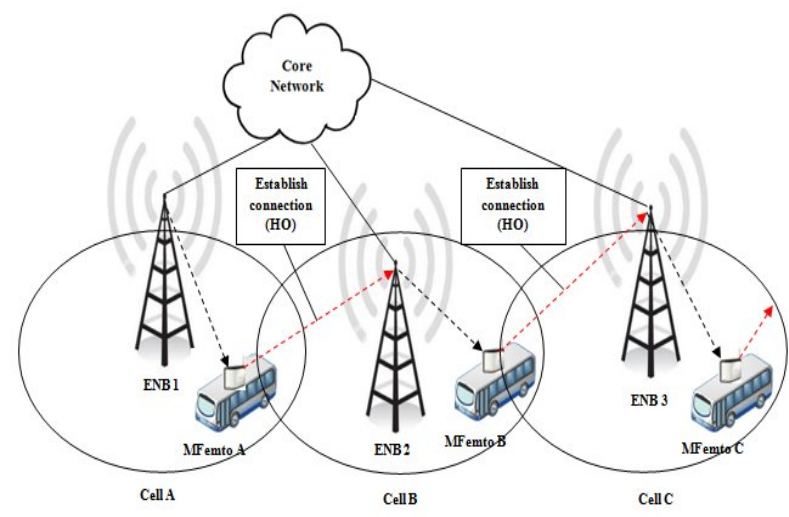

Fig.6.MFemto $\mathrm{HO}$ between two cells

However, in the above scenario there is always a chance of an overlapping issue between two MFemtos if e.g. MFemto handed over to cell $\mathrm{B}$ and there is not any other MFemto left cell $B$ at the same time due to the time delay issue. The problem with that is the connection of UEs who are inside the bus of MFemto will go down due to the fact that all the PRBs of the cell B are occupied and there is no chance for the MFemto to work at cell B. But there is another solution can be worked in this case and that leads to the following scenario (solution). Which means that those UE who are inside the bus/train can be served by any close by FFemtos or MFemtos even by a MFemto on another moving bus next to their bus/car and moving at the same speed of their bus/car.

Therefore, the following scenario considers as the worst case scenario which is concerning about the high traffic issue. For example, when there is a MFemto moving close to the cell edge and there are vehicular UEs inside their cars/buses near to the cell edge as well who are needed to be connected to a MFemto in order to maintain their connection. In this case, this connection of those vehicular UEs might not last more than few minutes depending on the speed of both; target MFemto and the vehicular UEs so as long the vehicular UEs move far away from the coverage area of the MFemto that they are attached to; the connection breaks down. Figure 7 illustrates this scenario in a diagram.

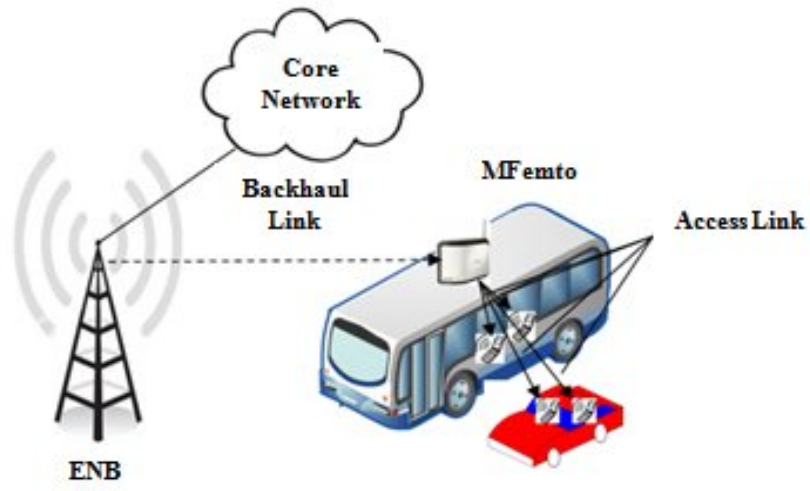

Fig.7.Connection establishment in the worst case traffic
Figure 8 shows that whenever there is a new vehicular/mobile UE needs to be attached to the MFemto; the Call Admission Control (CAC) initially checks whether the MFemto coverage is available or not. If the MFemto coverage is available, then the MFemto is the first choice to connect the UE to. The MFemto accepts the new UE if the received SINR level is satisfied and the PRBs in the MFemto are available since the SNIR ${ }_{\mathrm{TMF}}$ is the received SINR of the target MFemto. Then if the above conditions are not satisfied, the UE tries to connect with the overlaid macro-cellular network (eNB). The eNB does not allow the QoS degradation policy to accept any new UE and this UE will be rejected if the requested BW is not available in the overlaid macro-cellular network. The following figure illustrates the diagram that summarises the process that has been demonstrated earlier;

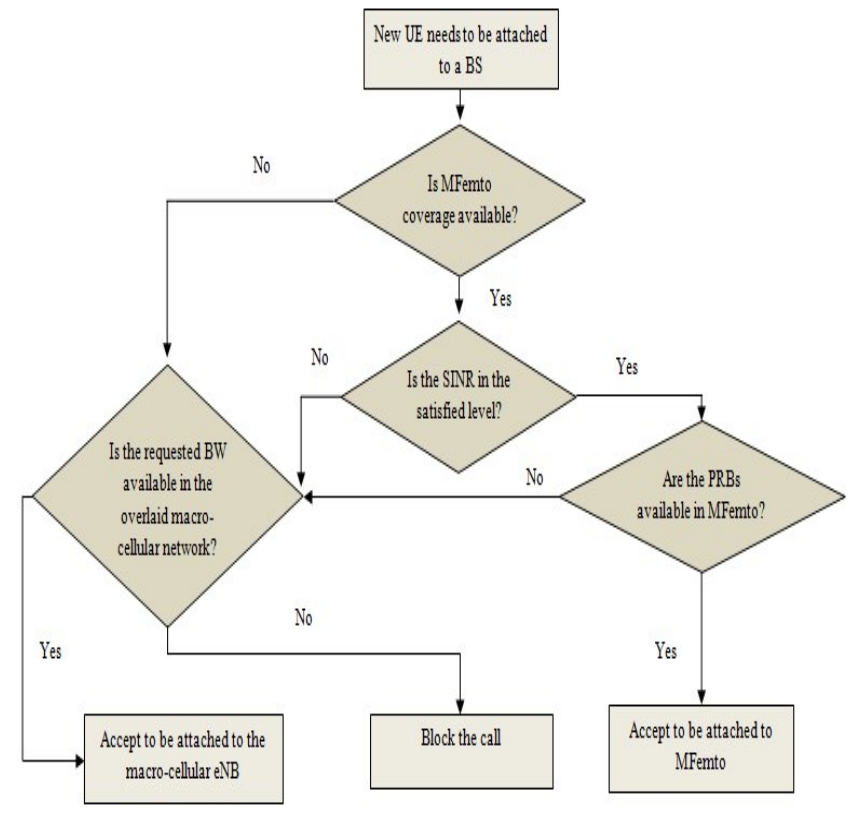

Fig.8.New connected UEs

\section{RESULTS AND DISCUSSION}

The MFemto/FFemto performance in LTE network has been evaluated using the dynamic level system simulator which considers the LTE specification. The MFemto/FFemto and mobile/fixed UEs were considered to be distributed independently in the cell. A single base station with three sites ( 3 eNBs) and 3 FFemtos or 1 MFemto in each $1 \mathrm{Km}^{2}$ have been considered. FFemto, MFemto and macrocell UEs were considered to be 6,8 and 40 respectively. The carrier bandwidth is fixed at $20 \mathrm{MHz}$ with $100 \mathrm{PRBs}$. In the eNB all UEs are equipped with a single antenna while in the FFemtos/MFemtos omnidirectonal antennas have been used. The gain on the backhaul link which is between the eNB and the MFemto $(\mathrm{G})$ is assumed to be $8 \mathrm{~dB}$. The following presents some of the simulated results of mobile UEs;

Figure 9 shows that the spectral efficiency of UEs who served by the MFemto is better than those UEs who served by the FFemto. Because controlling the coverage area and the 
interference of the MFemto is much easier than the FFemto. For example, sometimes when there is high interference between the MFemto and another MFemto or the eNB; it is easier to control the movement of that MFemto and let UEs enjoy better connection and services.

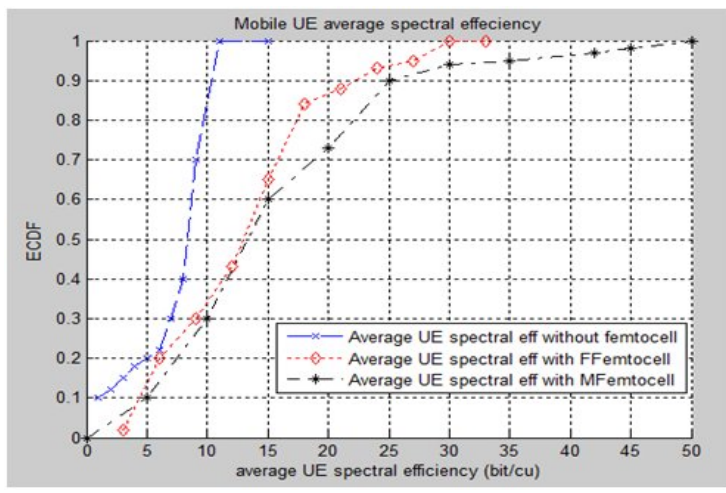

Fig.9. Spectral efficiency of mobile UEs without \& with FFemtos/MFemtos

While figure 10 is showing that the throughput of MFemto's UEs is much better than the FFemto's UEs and that due to the fact that the throughput can be affected by several factors as the following; packet loss due to the network congestion. Packets might be dropped when the packets queues are full due to the congestion or the packet might be lost due to the bit errors. Also, the UE throughput can be degraded when some users send large packets which require higher bandwidth than other users so the users who required more bandwidth will require more services and resources than others which expose the network to the extra load services. Those issues can be solved easily by implementing the MFemto in the network because using this technology serves the UEs faster with less power consumption. Also using MFemtos will relief the eNB from the extra load which might be in some areas that hard for the eNB to cover e.g. stadium.

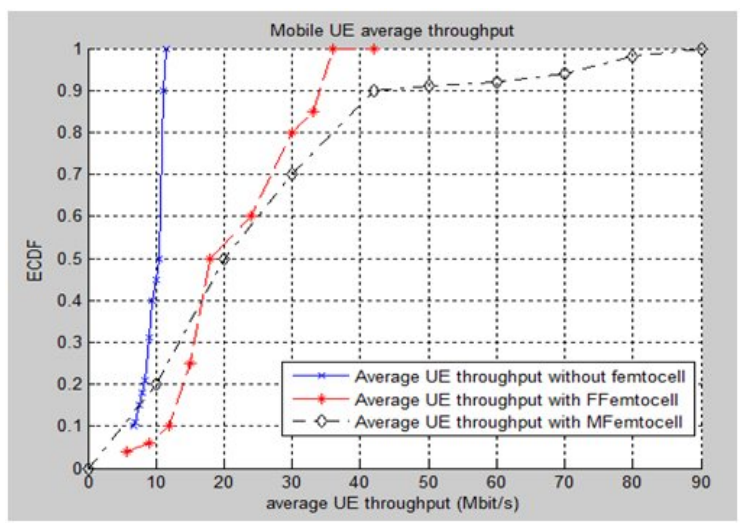

Fig.10. Throughput of mobile UEs without \& with FFemtos/MFemtos

Furthermore, the UE's SINR who is served by a MFemto starts almost at -20 and reach its max at 100 . Also, it is obvious that the SINR at range 30 intersect all together at ECDF 0.62 and that is due to the fact that the interference increases when the distance between the UE and the femtocell increases or the distance between the femtocell and the neighbouring femtocell or eNB decreases.

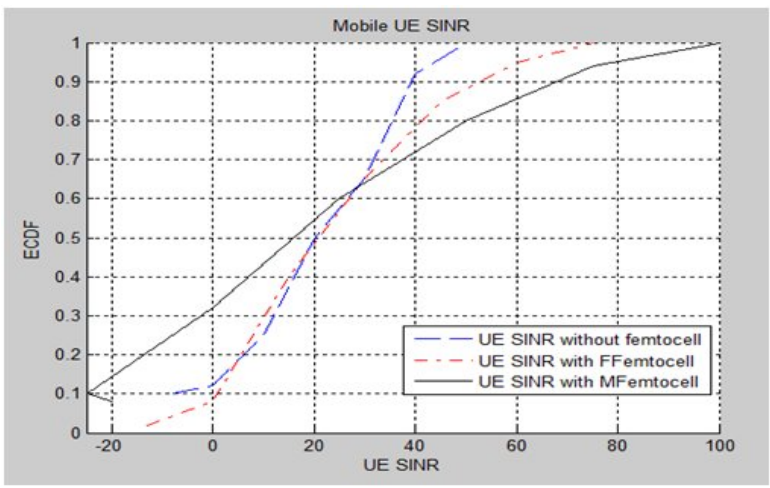

Fig.11. SINR of mobile UEs without \& with FFemtocells/MFemtocell

Since the $\mathrm{HO}$ procedure might be initiated either by the eNB/MFemto/FFemto or the UE; two HO strategies have been applied on $\mathrm{HO}$ scenarios which are the proactive and the reactive HO. Figure 12 shows that the performance of the reactive $\mathrm{HO}$ is much better that the proactive $\mathrm{HO}$ and that due to the fact that in the reactive $\mathrm{HO}$ strategy the $\mathrm{HO}$ is triggered only when the UE almost loses its connection with the serving eNB/MFemto/FFemto. This will decreases the number of HOs in the case of the reactive than the proactive $\mathrm{HO}$ which reduces the number of unnecessary HOs. Both of the reactive and proactive strategies have been applied on the studied scenarios in order to choose the best strategy that will give UEs higher performance than the normal HO. UEs in this case will have better performance and their connection will be maintained easily and quicker than the traditional way.

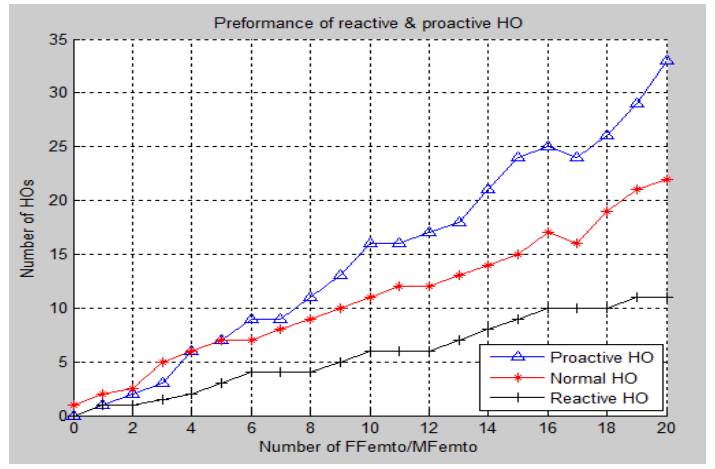

Fig.12.The proactive/reactive HO performance

The availability of distributing services among the limited number of PRBs and cell capacity are important elements to improve the overall system performance and handle the increased number of users and traffic. The load of the cell will be judged as a low, medium or high load depending on the services traffic. This traffic could be video streaming, VoIP, browsing, e-mailing, packets or data packets so the size of the traffic plays an important role in the load level. For example the number of the UEs in the cell might be low but the requested traffic is very high and each UE will occupied many PRBs that could be max of $10 \mathrm{RBs}$ while the number of UEs in 
another cell could be high but they use less traffic and less number of PRBs which could be min of 1 PRB per UE. In this simulation the data rate of video streaming, VoIP and Constant Bit Rate (CBR) were 242kbps, $12 \mathrm{kbps}$ and $100 \mathrm{kbps}$ respectively.

However, in the case of the low and medium load the results showed that there is not much difference in the UE throughput before and after adding the MFemto while in the case of the high load level the improvement in the UE throughput before and after adding the MFemto is obvious. In high load systems, adding MFemtos will be the solution to improve the QoS of cell edge UEs as well as the central UEs in the crowded areas (high loaded area). Figure 13 shows the system reaction under low, medium and high load traffic.

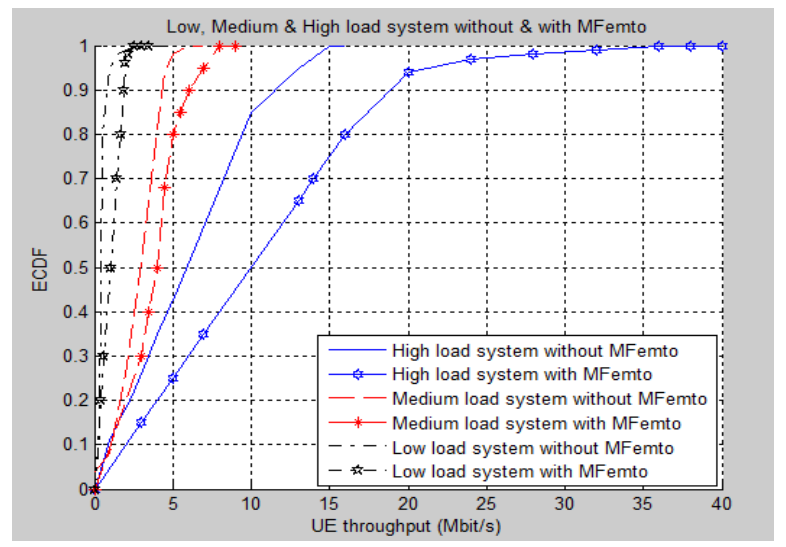

Fig.13.The system reaction under low, medium and high load

\section{CONCLUSION}

In this paper, a demonstration of two scenarios in LTE cellular network has been done which are; FFemtos with mobile UEs and MFemtos with mobile UEs. The performance of FFemtos and MFemtos has been investigated as well as the cell edge UEs QoS. It is obvious that the performance of the UEs has been improved after adding FFemtos and MFemtos. However, mobile UEs enjoyed better performance after adding the MFemtos since they can reach areas that FFemtos cannot reach.

The HO procedure of MFemto has been investigated since there is not much work done about this area in MFemto in LTE system. Three scenarios have been considered in this area which are; the HO from one MFemto to another and the second scenario is handing over the MFemto from one cell to another that leaded us to a third scenario which is considered as a worst case traffic scenario. In order to improve the performance of $\mathrm{HO}$ procedure in FFemto/MFemto, two $\mathrm{HO}$ strategies are needed to be considered in the future work; the proactive and reactive $\mathrm{HO}$ strategies.

Since the availability of PRBs and cell capacity play an important role in improving the system performance and handling the increased number of UEs, this study has studies the system behavior under the low, medium and high loads traffic. The achieved results showed that adding the MFemto in the case of the high load traffic will improve the UEs throughput since it reliefs the eNB from the extra load traffic to let UEs enjoy better QoS.

\section{ACKNOWLEDGMENT}

The authors would like to thank all those who contribute to the completion and success of this work. Also, the science and technology department at Middlesex University played a significant role in supporting and backing this work till it completes.

\section{REFERENCES}

[1] O. Oyman, N. Laneman and S. Sandhu, "Multihop relaying for broadband wireless mesh networks: from theory to practice", IEEE Commun. Magazine, vol. 45, no. 11, pp. 116-122, Nov.2007.

[2] C.X. Wang, X. Hong, X. Cheng, G. Zhang, and J. Thompson "Cooperative MIMO channel models: a survey", IEEE Commum. Magazine, vol. 48, no. 2, pp.80-87, Feb. 2010.

[3] X. Hong, C.-X. Wang, M. Uysal, X. Ge, and S. Ouyang, "Capacity analysis of hybrid cognitive radio networks with distributed VAAs," IEEE Trans. Veh. Techno., vol. 59, no. 7, pp. 3510-3523, Sept. 2010.

[4] O. Oyman, Opportunistic scheduling and spectrum reuse in relaybasedcellular networks,'IEEE Trans. Wireless Commun., vol. 9, no. 3, pp.1074-1085, Mar. 2010.

[5] G. Harikumar, "Femtocells: implementation challenges and solutions", Director, CDMA Femtocell program, Airvana Incorporated, IEEE Globecomm., Dec 2008.

[6] L. Xiao, T. Fuja, and D. Costello, "An analysis of mobile relaying forcoverage extension," inProc. IEEE Symp.Info. Theory, July 2008, pp. 2262-2266.

[7] G. de la Roche, A. Valcarce and J. Zhang, "Hybrid Model for Indoor-toOutdoor Femtocell Radio Coverage Prediction", Centre for Wireless Network design, University of Bedfordshire, Luton, UK, IEEE, 2011.

[8] S. Al-Rubaye, "Radio Network management in Cognitive LTE/Femtocell Systems", A Ph.D thesis, Electronic and Computer Engineering, School of Engineering and Design, Brunel University, Sep 2012.

[9] Y. Sui, J. Vihriala, A. Papadogiannis \& M. Sternad, "Moving Cells: A promising Solution to Boost Performance for Vehicular Users", Chalmers University of Technology, 2013.

[10] M. Bennis, L. Giupponi, E.M Diaz \& M. Lalam, "Interference management in self-organized femtocell networks: The BeFEMTO approach", Wireless Communication, Vehicular Technology, Information Theory and Aerospace \& Electronics Systems Technology (Wireless VITAE), $20112^{\text {nd }}$ International Conference, 2011.

[11] M. Thiago Martins de, B. Gerhard \& S. Eiko, "QoS-aware Scheduling for In-Band Relays in LTE-Advanced", Systems, Communication and Coding (SCC) Proceeding of $20139^{\text {th }}$ International IGT Conference, 2013.

[12] P. Vinh Van, K. Horneman, Y. Ling \& J. Vihriala, "Providing enhanced cellular coverage in public transportation with smart relay systems", Vehicular Networking Conference (VNC), 2010 IEEE, 2010.

[13] S. Ranvier, "Path loss models", S-72.333 Physical layer methods in wireless communication systems, 2004.

[14] TELETOPIX.ORG, "Penetration Loss in LTE", Telecom Techniques Guide, 2013.

[15] J. Hamalainen, "Femtocells: Technology and Developments", Wireless Information Theory Summer School, Center for Wireless Communications, Aalto University, 2011

[16] R.Raheem, A.Lasebae, M.Aiash, J.Loo "From Fixed to Mobile Femtocells inLTE systems: Issues and Challenges", Second International Conference on Future Generation Communication Technologies (FGCT 2013), Middlesex University, London, UK, 2013. 